\title{
Effective Crystal Field for Trivalent First Transition Row Ions
}

\author{
A. V. SINITSKY, ${ }^{1,2}$ M. B. DARHOVSKII, ${ }^{1,2}$ A. L. TCHOUGRÉEFF, ${ }^{1,2}$ \\ I. A. MISURKIN ${ }^{\mathbf{1}}$ \\ ${ }^{1}$ Karpov Institute of Physical Chemistry, Vorontsovo Pole 10, 103064 Moscow, Russia \\ ${ }^{2}$ Center for Computational Chemistry at the M.V. Keldysh Institute for Applied Mathematics RAS, \\ 125047 Moscow, Russia
}

Received 18 July 2001; accepted 22 December 2001

DOI 10.1002/qua.10174

\begin{abstract}
In the present work the semiempirical effective crystal field (ECF) method previously designed for electronic structure calculations of transition metal complexes and utilizing non-Hartree-Fock trial wave function and parameterized for complexes of doubly charged $\mathrm{Cr}^{2+}, \mathrm{V}^{2+}, \mathrm{Mn}^{2+}, \mathrm{Fe}^{2+}, \mathrm{Co}^{2+}$, and $\mathrm{Ni}^{2+}$ cations is extended to complexes of triply charged cations of $3 d$-elements. With the parameters adjusted the ECF method is applied to calculations of ground states and low-energy spectra of the $d$-shells of fluoro-, chloro-, aqua-, amino-, and cyano-complexes of the triply charged cations. Obtained total spin and symmetry of the ground states match the experimentally observed ones. Satisfactory agreement between the calculated and experimental $d$-shell electronic transition energies is achieved as well. (C) 2002 Wiley Periodicals, Inc. Int J Quantum Chem 88: 370-379, 2002
\end{abstract}

Key words: effective crystal field method; transition metal complexes; trivalent ions

\section{Introduction}

C alculations on magnetic and optical properties of transition metal complexes (TMCs) are important problems of theoretical chemistry. Semiempirical [1-5] as well as ab initio methods [6-13] were applied for that, both substantially using the Hartree-Fock-Roothaan [or the self-consistent field (SCF) molecular orbital (MO)-LCAO] approximation. The $a b$ initio calculations are highly

Correspondence to: A. L. Tchougréeff; e-mail: andrei@cc. nifhi.ac.ru time consuming when applied to TMCs. The reason is a huge number of electronic states to be included in the configuration interaction $(\mathrm{CI})$ procedure, due to poor convergence of the $\mathrm{CI}$ series when the canonical Hartree-Fock (MOs) are used for constructing the configurations. Thus, $a b$ initio calculations can be performed only for relatively small systems, for which nevertheless a considerable agreement of calculated and experimental transition energies can be obtained [11, 12]. Semiempirical methods based on the SCF approximation are less demanding for computational resources, although results obtained strongly depend on parameterization of numerous molecular integrals. 
The application of semiempirical and $a b$ initio methods based on Hartree-Fock approximation to the TMC electronic structure calculations is, however, complicated by violation of the SCF approximation itself. This is exhibited in some of its important consequences [14]. Namely, the Aufbau principle and Koopman's theorem are not fulfilled for the MOs with considerable weight of the atomic $d$-orbitals; the relaxation energy for the latter (the difference between the ionization potential calculated by the Koopman's theorem and its experimental value) can achieve 10 or even $20 \mathrm{eV}$ for ionization from these MOs, and the MOs being higher in energy can turn out to be occupied while MOs of lower energy remain vacant or singly occupied. Also, the SCF iteration process often converges slowly or oscillates.

All these observations indicate that behavior of $d$-electrons in the TMC goes beyond the SCF approximation's frames, which can be characterized as a motion of independent electrons in the SCF induced by nuclear cores and other electrons. In contrast, $d$-electrons in TMCs are strongly correlated (as compared to those in the ligand orbitals) and form a well-localized separate group. As ground-state spin and low-energy excitations of TMCs mainly depend on $d$-electrons' state [15], accounting for correlations of the latter is of principal importance for the description of magnetic and optical properties of the TMCs.

For interpreting experimental data and explaining properties of TMCs the phenomenological crystal field theory (CFT) $[15,16]$ is widely used. The latter describes the TMCs in terms of excitations of their $d$-shells only. According to the CFT the oneelectron states in the $d$-shells are split by an electrostatic field induced by effective charges residing in the ligands. The main defect of the CFT is lack of details of the ligand electronic structure that entails the limitation of the interaction between the $d$-shell of the central atom and the ligands to the purely electrostatic one. For that reason, the $d$-level splitting parameter $10 \mathrm{Dq}$ is essentially underestimated and the one-electron splitting parameters cannot be calculated within the CFT itself, and remain independent parameters of the theory. The ligand field theory (LFT) $[15,16]$ partially takes into account the covalent character of interactions between the ligands and the central ion. However, the LFT calculations are in fact equivalent to the Hartree-Fock approximation and reduce to taking into account the MOs symmetry when making linear combinations with the $d$-orbitals. For this reason, splitting characteristics calculated within the model are different from ones fitting in experimental data analysis.

For taking into account in the semiempirical realm qualitative features induced by both electron correlation and covalency effects and circumventing the problems of the original naive CFT and LFT, the effective crystal field (ECF) method was proposed [14], which is based on a combination of the effective Hamiltonian method and the group function technique $[17,18]$. Basic features and formulae of this method are given below.

\section{Effective Crystal Field Method}

The trial wave function $\Phi$ of electrons in TMC has the form of the antisymmetrized product of the full CI wave function for $d$-electrons $\Psi_{M}$, and the single determinant wave function $\Psi_{L}$ for remaining electrons of the TMC:

$$
\Phi=\Psi_{M}\left(n_{d}\right) \wedge \Psi_{L}\left(n_{l}\right) .
$$

Such a function is a special case of the group function [18] approximation where the groups are formed by electrons in the $d$-shell and those in other orbitals, respectively. This form of the wave function allows the use of the difference of the characteristic values of interaction parameters in the $d$ shell and in the ligands for taking into account electron correlations at different levels.

To arrive at the above form of the trial wave function, we note that the general form of the exact wave function can be presented as a linear combination of the functions having all possible electron distributions between the two subsystems singled out. However, in Ref. [14] it was shown that the wave function Eq. (1) with a fixed number $n_{d}$ of electrons in the $d$-shell, which is equal to their number in the ground state of the corresponding free metal ion, must be obtained from the mentioned linear combination by projecting onto subspace spanned by the functions of the form Eq. (1).

Within the ECF theory the total Hamiltonian is rewritten in the form

$$
H=H_{d}+H_{l}+H_{c}+H_{r}
$$

where $H_{d}$ is the Hamiltonian for $d$-electrons in the field of the TMC atomic cores, $H_{l}$ is the Hamiltonian for the ligand subsystem electrons, and $H_{c}$ and $H_{r}$ 
are, respectively, the operators of Coulomb and resonance interactions between two subsystems of the TMC.

For the charge transfer states, the Löwdin partition technique [17] as a version of effective Hamiltonian method was used in Ref. [14]. After projecting the exact Hamiltonian, Eq. (2) is replaced by the effective one acting in the subspace spanned by the functions with the fixed number $n_{d}$ of $d$-electrons. Eigenvalues of the effective Hamiltonian coincide with exact Hamiltonian eigenvalues by construction. By simple algebra the explicit form of the effective Hamiltonian is obtained [14]:

$$
\begin{aligned}
H^{\mathrm{eff}} & =P H_{0} P+H_{r r} \\
H_{0} & =H_{d}+H_{l}+H_{c} \\
H_{r r} & =P H_{r} Q\left(E Q-Q H_{0} Q\right)^{-1} Q H_{r} P
\end{aligned}
$$

where $P$ is the operator projecting to the subspace of the functions with the fixed number $n_{d}$ of the $d$-shell electrons, $n_{l}=N-n_{d}$ is that of the ligand subsystem electrons, and $Q=1-P$.

The TMC eigenstate energies must be obtained from the relation

$$
E_{n}=\left\langle\Phi_{n}\left|H^{\text {eff }}\left(E_{n}\right)\right| \Phi_{n}\right\rangle .
$$

Because the effective Hamiltonian depends on energy, the last equation must be solved iteratively until convergence in energy is achieved. However, because the charge transfer states (determining the poles of the above resolvent term) lay significantly higher in energy than the $d$-shell excitations this dependence turns out to be weak and can be neglected, so one can set

$$
H^{\mathrm{eff}}\left(E_{n}\right) \approx H^{\mathrm{eff}}\left(E_{0}\right),
$$

where $E_{0}$ is the ground-state energy of the Hamiltonian $H_{0}$. Thus, the obtained effective Hamiltonian corresponds to the second order of the RaleighSchrödinger perturbation theory in $H_{r}$. Variation principle applied to the effective Hamiltonian with the trial function of the above form leads to the self-consistent system of equations:

$$
\begin{aligned}
H_{d}^{\mathrm{eff}} \Phi_{n}^{d} & =E_{n}^{d} \Phi_{n}^{d} \\
H_{l}^{\mathrm{eff}} \Phi^{l} & =E^{l} \Phi^{l} \\
H_{d}^{\mathrm{eff}} & =H_{d}+\left\langle\Phi^{l}\left|H_{c}+H_{r r}\right| \Phi^{l}\right\rangle
\end{aligned}
$$

$$
H_{l}^{\text {eff }}=H_{l}+\left\langle\Phi_{n}^{d}\left|H_{c}+H_{r r}\right| \Phi_{n}^{d}\right\rangle .
$$

In the above system, the effective Hamiltonian $H_{d}^{\text {eff }}$ for the $d$-electron subsystem depends on the wave function of the ligand subsystem $\Phi^{l}$, and in turn the effective Hamiltonian $H_{l}^{\text {eff }}$ for the ligand subsystem depends on the $d$-electrons' wave functions $\Phi_{n}^{d}$. These equations must be solved self-consistently as well. In the ECF method [14], the Slater determinant $\Phi^{l}$ is constructed of MOs of the ligand subsystem, obtained from the Hartree-Fock equations in the $\mathrm{CNDO} / 2$ approximation for the valence electrons of the ligands. In this case the transition from the bare Hamiltonian $H_{l}^{\text {eff }}$ for the ligand subsystem to the corresponding effective (dressed) Hamiltonian reduces to renormalization of oneelectron parameters related to the transition metal ion:

$$
\begin{aligned}
& U_{i i}^{\text {eff }}=U_{i i}+\frac{1}{5} n_{d} \sum_{\mu} g_{\mu i} \\
& Z_{M}^{\text {eff }}=Z_{M}-n_{d}
\end{aligned}
$$

where $U_{i i}$ is the parameter of the interaction of $4 s-$ and $4 p$-electrons $\left(i=4 s, 4 p_{x}, 4 p_{y}, 4 p_{z}\right)$ with the metal core, $Z_{M}$ is metal core charge, and $g_{\mu i}$ are the Oleari parameters of intraatomic Coulomb interactions. The $\Phi^{l}$ function thus obtained is used further for constructing the effective Hamiltonian for the $d$ shell. Diagonalization of the latter gives both the wave functions of the $d$-shell and the $d$-electron state energies. The effective Hamiltonian for the $d$-shell after averaging Eq. (6) intersubsystem interaction operators $H_{c}$ and $H_{r r}$ over the ground state of the ligand system takes the form

$$
H_{d}^{\mathrm{eff}}=\sum_{\mu \nu \sigma} U_{\mu \nu}^{\mathrm{eff}} d_{\mu \sigma}^{+} d_{\nu \sigma}+\frac{1}{2} \sum_{\mu \nu \rho \eta} \sum_{\sigma \tau}(\mu \nu \mid \rho \eta) d_{\mu \sigma}^{+} d_{\rho \tau}^{+} d_{\eta \tau} d_{\nu \sigma r}
$$

where $d_{\mu \sigma}^{+}\left(d_{\nu \sigma}\right)$ are operators of creation (annihilation) of electron with the spin projection $\sigma$ on the $\mu$ th $d$-atomic orbital (AO) and $(\mu \nu \mid \rho \eta)$ are the twoelectron integrals of the Coulomb interaction in the $d$-shell. Effective one-electron parameters $U_{\mu \nu}^{\text {eff }}$ of the $d$-shell contain contributions from the Coulomb and from the projected [Eq. (5)] resonance interaction with the ligand subsystem:

$$
U_{\mu \nu}^{\mathrm{eff}}=\delta_{\mu \nu} U_{d d}+W_{\mu \nu}^{\mathrm{atom}}+W_{\mu \nu}^{\mathrm{ion}}+W_{\mu \nu}^{\mathrm{cov}}
$$


where

$$
\begin{aligned}
W_{\mu \nu}^{\mathrm{atom}} & =\delta_{\mu \nu}\left(\sum_{i \in s, p} g_{\mu i} P_{i i}\right) \\
W_{\mu \nu}^{\mathrm{ion}} & =\sum_{l}\left(P_{l l}-Z_{l}\right) V_{\mu \nu}^{l} .
\end{aligned}
$$

Here $P_{i i}$ is the one-electron density matrix element for the ligand subsystem, $P_{l l}=\sum_{i \in l} P_{i i}$, $Z_{l}$ is the $l$ th atom core charge, and $V_{\mu \nu}^{l}$ is the matrix element of the $d$-electron potential energy in the electrostatic field of a unit point charge placed on the lth ligand atom. The covalence contribution to the ECF is given by

$$
W_{\mu \nu}^{\mathrm{cov}}=-\sum_{i}^{(\mathrm{MO})} \beta_{\mu i} \beta_{\nu i}\left\{\frac{\left(1-n_{i}\right)^{2}}{\Delta E_{d i}}-\frac{n_{i}^{2}}{\Delta E_{i d}}\right\},
$$

where $\beta_{\mu i}$ is the resonance integral between the $\mu$ th $d$-orbital and the $j$ th ligand $\mathrm{MO}, n_{j}(=0,1)$ is the occupation number of the $j$ th $\mathrm{MO}$, and $\Delta E_{d i}\left(\Delta E_{i d}\right)$ are excitation energies required to transfer an electron from the $d$-shell (ith $\mathrm{MO}$ ) to the $i$ th $\mathrm{MO}(d$ shell). The intersubsystem's resonance integrals are calculated by the equation

$$
\beta_{\mu k}=\left(I_{d}+I_{k}\right) S_{\mu k} \beta^{M-L},
$$

where $I_{d}$ and $I_{k}$ are valence ionization potentials of the $d$-shell and of the $k$ th $\mathrm{AO}$ in the ligand subsystem, respectively, $S_{\mu k}$ is the AO's overlap integral, and $\beta^{M-L}$ is a dimensionless parameter scaling the resonance interaction between the $d$-shell and a ligand atom. Charge transfer energies $\Delta E_{d i}$ and $\Delta E_{i d}$ are estimated according to

$$
\begin{aligned}
& \Delta E_{d i}=I_{d}+\epsilon_{i}-G_{d i} \\
& \Delta E_{i d}=-A_{d}-\epsilon_{i}-G_{d i},
\end{aligned}
$$

where $\epsilon_{i}$ is the $i$ th MO energy, $G_{d i}$ is the Coulomb interaction energy between an electron and a hole localized in the $d$-shell and on the $i$ th MO, respectively, and $I_{d}$ and $A_{d}$ are, respectively, the ionization potential and electron affinity of the $d$-shell.

The effective Hamiltonian for the $d$-shell Eq. (8) formally coincides with the CFT Hamiltonian. The substantial difference is the covalence contribution Eq. (11) to the $d$-shell one-electron parameters Eq. (9), taking into account the effect of virtual charge transfers between the metal $d$-shell and the ligands. Thereby, the ECF contains not only electrostatic but also covalence terms coming from the resonance interactions between the $d$-shell and the ligands.

According to calculations performed in Refs. [14] and [19-22] for the TMCs of divalent cations, the covalence contribution to the splitting parameter $10 \mathrm{Dq}$ dominates and gives up to $90 \%$ of the total. This stresses the importance of the procedures described above aimed to include the covalence interaction in an economic and transparent fashion into the effective Hamiltonian parameters. The parameters of the ECF method for the complexes of divalent cations of the first transition range metals have been found, tested, and employed in Refs. [14] and [19-22]. They allowed the correct description of the symmetry of the ground states and optical $d-d$ transition energies with precision up to $1000 \mathrm{~cm}^{-1}$ for about 100 of the TMCs of the first transition row divalent cations ranging from hexafluoroanions to porphyrine complexes. Therefore, the use of the ECF allows significant improvement of semiempirical description of TMC electronic structure.

\section{Results and Discussion}

The purpose of the present work was to explore the possibility to extend the parameterization of Refs. [14] and [19-22] to the complexes of trivalent ions of the first transition row. Note that when talking about di- and trivalent cations we imply the Werner complexes for which the $d$-electron subsystem contains the same number of electrons as the $3 d$-shell of the corresponding isolated cation, for example, the $d$-electron subsystem of the $\mathrm{Fe}^{3+}$ complexes has $3 d^{5}$ configuration with five $d$-electrons.

The whole set of the parameters of the ECF method consists of those of the CNDO/2 method for the ligand atoms, specific parameters $U_{d d}, \zeta_{d}, B$, and $C$ (see below) for the $d$-shell, and Oleari parameters describing the intraatomic part of the Coulomb interaction between subsystems. In this article only the core attraction of $d$-electrons $U_{d d}$ for each metal ion and the $\beta^{M-L}$ resonance parameters for each pair metal $(M)$-donor atom $(L)$ have been fitted. The $\beta^{M-L}$ parameters have been adjusted to fit the calculated excitation energies to the experimental ones for the complexes with organic amines, pyridine and its derivatives, and other nitrogenand oxygen-containing, ligands, halogen, anions, and so forth. 
TABLE I

Covalent and ionic contributions to the $d$-level crystal field splitting parameter $10 D q$ in octahedral complexes of trivalent cations $\left(\mathrm{cm}^{-1}\right)$.

\begin{tabular}{|c|c|c|c|c|}
\hline M & $10 D q^{\text {ion }}$ & $10 D q^{\mathrm{cov}}$ & $10 D q^{\text {total }}$ & $10 D q^{\operatorname{expt}}[23,24]$ \\
\hline \multicolumn{5}{|c|}{$\left[\mathrm{MF}_{6}\right]^{3-}$} \\
\hline V & 2769 & 13304 & 16073 & 15900 \\
\hline $\mathrm{Cr}$ & 2388 & 12941 & 15329 & 15200 \\
\hline $\mathrm{Fe}$ & 1330 & 12580 & 13910 & 13900 \\
\hline Co & 885 & 12229 & 13114 & 13100 \\
\hline \multicolumn{5}{|c|}{$\left[\mathrm{MCl}_{6}\right]^{3-}$} \\
\hline $\mathrm{Cr}$ & 646 & 12560 & 13206 & 13800 \\
\hline $\mathrm{Mn}$ & 565 & 16987 & 17552 & 17540 \\
\hline $\mathrm{Fe}\left(\left[\mathrm{FeCl}_{4}\right]^{-}\right)$ & 263 & 4721 & 4984 & 5000 \\
\hline \multicolumn{5}{|c|}{$\left[\mathrm{M}\left(\mathrm{H}_{2} \mathrm{O}\right)_{6}\right]^{3+}$} \\
\hline V & 1088 & 16512 & 17600 & 18400 \\
\hline $\mathrm{Cr}$ & 769 & 16638 & 17407 & 17390 \\
\hline $\mathrm{Fe}$ & 1329 & 12621 & 13950 & 14200 \\
\hline Co & 221 & 17936 & 18157 & 18160 \\
\hline \multicolumn{5}{|c|}{$\left[\mathrm{M}\left(\mathrm{NH}_{3}\right)_{6}\right]^{3+}$} \\
\hline $\mathrm{Cr}$ & 554 & 20984 & 21538 & 21550 \\
\hline Co & 343 & 22532 & 22875 & 22870 \\
\hline \multicolumn{5}{|c|}{$\left[\mathrm{M}(\mathrm{CN})_{6}\right]^{3-}$} \\
\hline $\mathrm{Cr}$ & 772 & 26061 & 26833 & 26770 \\
\hline $\mathrm{Fe}$ & 603 & 34401 & 35004 & 35000 \\
\hline Co & 518 & 32075 & 32593 & 32200 \\
\hline
\end{tabular}

In this article the ECF calculations on octahedral complexes of trivalent cations of the first transition row have been performed. We considered only those complexes whose both geometry and transition energies and the corresponding symmetries of the excited states are known, namely, $\mathrm{V}^{3+}, \mathrm{Cr}^{3+}$, $\mathrm{Mn}^{3+}, \mathrm{Fe}^{3+}$, and $\mathrm{Co}^{3+}$. (The data on $\mathrm{Ni}^{3+}$ and $\mathrm{Cu}^{3+}$ complexes are not readily available because the latter are largely unstable.) The geometry structure parameters-the atomic coordinates, bond lengths, and valence angles-were taken from the literature (the corresponding references are given in the tables).

The $U_{d d}$ and $\beta^{M-L}$ parameters determining the value of one-electron splitting parameter $10 D q$ in the $d$-shell and the spectrum of the $d$-d-transitions were fitted so that the best agreement of calculated and experimental values of $10 D q$ and of the excitation energies is achieved. The values of $U_{d d}$ for each metal of the considered $\mathrm{V}, \mathrm{Cr}, \mathrm{Fe}$, and $\mathrm{Co}$ series were modified so that the positive values of $\Delta E_{d j}$ and $\Delta E_{j d}$ Eq. (13) are guaranteed to ensure the stability of the system with respect to electron transfer between the subsystems. After that the $\beta^{M-L}$ parameters have been fitted to reach a required agreement between the calculated and experimental values of the splitting parameters and calculated and experimental excitation energies.

The calculated $10 D q$ values, the ionic and covalent contributions to them, and the respective experimental values are given in Table I. The fitted values of the parameters $U_{d d}$ and $\beta^{M-L}$ for the trivalent ions, the respective parameters for the divalent ions, and also bond length differences between the di- and trivalent cations complexes (in those cases when the corresponding data for both complexes are available) are given. Also the Slater exponents for the valence $4 s^{-}, 4 p$-, and $3 d$-orbitals of the trivalent ions are given. These quantities, chosen in accordance with the Burns rules [25] accepted in the 
TABLE II

Parameters $\beta^{M-L}, U_{d d}(\mathrm{eV})$, Slater exponents for the valence $4 s-$, $4 p-$, and $3 d$-orbitals and difference of metal-donor atom separations for complexes of doubly and triply charged transition metal cations.

\begin{tabular}{|c|c|c|c|c|c|c|}
\hline & & V & $\mathrm{Cr}$ & $\mathrm{Mn}$ & $\mathrm{Fe}$ & Co \\
\hline \multirow[t]{2}{*}{$\mathrm{F}$} & 2 & - & - & 1.225 & 1.431 & 1.826 \\
\hline & 3 & 1.051 & 1.825 & - & 1.244 & 1.664 \\
\hline$\Delta r_{23}$ & & - & - & - & 0.308 & 0.1444 \\
\hline \multirow[t]{2}{*}{$\mathrm{Cl}$} & 2 & 0.975 & - & 1.250 & 1.339 & 1.588 \\
\hline & 3 & - & 1.020 & 0.765 & $1.60\left(\left[\mathrm{MCl}_{4}\right]^{-}\right)$ & - \\
\hline$\Delta r_{23}$ & & - & - & 0.228 & 0.059 & - \\
\hline \multirow[t]{2}{*}{$\mathrm{O}^{20}$} & 2 & 0.985 & 1.400 & 1.325 & 1.825 & 1.838 \\
\hline & 3 & 1.180 & 1.004 & - & 1.484 & 2.540 \\
\hline$\Delta r_{23}$ & & 0.133 & 0.153 & - & 0.14 & 0.011 \\
\hline \multirow[t]{2}{*}{$N^{20}$} & 2 & - & - & - & 1.505 & 1.505 \\
\hline & 3 & - & 1.002 & - & - & 1.470 \\
\hline$\Delta r_{23}$ & & - & - & - & - & 0.146 \\
\hline \multirow[t]{2}{*}{$\mathrm{C}^{20}$} & 2 & - & - & - & 1.263 & - \\
\hline & 3 & - & 0.728 & - & 0.995 & 0.940 \\
\hline$\Delta r_{23}$ & & - & - & - & -0.037 & - \\
\hline \multirow[t]{2}{*}{$U_{d d}$} & 2 & -56.42 & -73.04 & -92.49 & -107.49 & -129.79 \\
\hline & 3 & -47.0 & -70.0 & -90.0 & -105.0 & -125.0 \\
\hline \multirow{2}{*}{$\zeta_{s}$} & 2 & 1.200 & 1.325 & 1.450 & 1.575 & 1.700 \\
\hline & 3 & 1.325 & 1.450 & 1.575 & 1.700 & 1.825 \\
\hline \multirow[t]{2}{*}{$\zeta_{p}$} & 2 & 0.750 & 0.825 & 0.900 & 0.975 & 1.050 \\
\hline & 3 & 0.825 & 0.900 & 0.975 & 1.050 & 1.125 \\
\hline \multirow[t]{2}{*}{$\zeta_{d}$} & 2 & 2.501 & 2.650 & 2.935 & 3.152 & 3.369 \\
\hline & 3 & 2.650 & 2.935 & 3.152 & 3.369 & 3.586 \\
\hline
\end{tabular}

$$
\Delta r_{23}=r\left(\mathrm{M}^{2+}-\mathrm{L}\right)-r\left(\mathrm{M}^{3+}-\mathrm{L}\right),((\AA))
$$

original ECF method [14], are less diffuse than the respective orbitals of the divalent ions. It should be noted that the $U_{d d}$ values for the trivalent ions are smaller (by the absolute value) by only a few electronvolts (or a few percent) than the values of the same parameters employed previously to describe their divalent analogs. The parameters $\beta^{M-L}$ for the trivalent cations are as a rule smaller than those for the divalent ones with the exception of hexahydrate complexes of $\mathrm{V}^{3+}$ and $\mathrm{Co}^{3+}$. One also may note that the values $\beta^{M-L}$ for all the donor atoms $L$ are systematically smaller for the trivalent ions but increase with the atomic number of the metal as for the divalent ions. Because we considered only one complex of $\mathrm{Mn}$, we had to take the $U_{d d}$ value by a few percent smaller than the absolute value for the divalent manganese ion by analogy with $\mathrm{Cr}, \mathrm{Fe}$, and Co.

We note also that the fitted values of the parameters are close to the respective values for the divalent ions, an additional proof of consistency of the ECF method permitting a comparatively simple extension to a new class of objects-complexes of trivalent cations. Covalent interactions in the considered complexes of the trivalent cations contribute about $80 \%$ to the $d$-states splitting as in the case of the divalent cation complexes.

In Tables II-VI the results of calculations on octahedral complexes of the transition metal trivalent cations are given. Such complexes as $\left[\mathrm{VF}_{6}\right]^{3+}$, $\left[\mathrm{V}\left(\mathrm{H}_{2} \mathrm{O}\right)_{6}\right]^{3+}$, and $\left[\mathrm{CoF}_{6}\right]^{3+}$ are the Jahn-Teller complexes. Our assumption that these complexes possess an octahedral symmetry adds an additional uncertainty to the calculated transition energies, so one may expect somewhat a larger difference between the calculated and experimental transition energies for these complexes.

Values of the Racah parameters $B$ and $C$ describing the electron interaction in the $d$-shell cited in the literature were used in our calculations. But, where such data were absent in the original works we used the Racah parameters given in the tables in Ref. [15].

Despite all the sources of uncertainty mentioned above, we succeeded in fitting the $\beta^{M-L}$ parameters so that the correct ground-state term symmetry and 
TABLE III

Calculated and experimental excitation energies $\left(\mathrm{cm}^{-1}\right)$ of transition metal fluorocomplexes.

\begin{tabular}{|c|c|c|}
\hline Transition & $\begin{array}{c}\mathrm{E} \\
\text { (theor) }\end{array}$ & $E(\exp )$ \\
\hline \multicolumn{3}{|c|}{$\begin{array}{c}{\left[\mathrm{VF}_{6}\right]^{3-}, \text { ground state }{ }^{3} T_{1 g}[23,26]} \\
\mathrm{B}=648, \mathrm{C}=3815\end{array}$} \\
\hline${ }^{3} T_{1 g} \rightarrow{ }^{1} T_{2 g}$ & 9225 & 10200 \\
\hline$\rightarrow{ }^{1} E_{g}$ & 9374 & 10200 \\
\hline$\rightarrow{ }^{3} T_{2 g}$ & 16098 & 14800 \\
\hline$\rightarrow{ }^{1} A_{1 g}$ & 20279 & - \\
\hline$\rightarrow{ }^{3} T_{1 g}$ & 24508 & 23000 \\
\hline$\rightarrow{ }^{1} T_{2 g}$ & 26578 & - \\
\hline$\rightarrow{ }^{1} T_{1 g}$ & 28874 & - \\
\hline \multicolumn{3}{|c|}{$\begin{array}{c}{\left[\mathrm{CrF}_{6}\right]^{3-}, \text { ground state }{ }^{4} A_{2 g}[27,28]} \\
B=643, \mathrm{C}=4133\end{array}$} \\
\hline${ }^{4} A_{2 g} \rightarrow{ }^{2} E_{g}$ & 10980 & - \\
\hline$\rightarrow{ }^{2} T_{1 g}$ & 11597 & - \\
\hline$\rightarrow{ }^{4} T_{2 g}$ & 15329 & 15200 \\
\hline$\rightarrow{ }^{2} T_{2 g}$ & 16778 & - \\
\hline$\rightarrow{ }^{4} T_{1 g}$ & 21680 & 21800 \\
\hline$\rightarrow{ }^{2} A_{1 g}$ & 24201 & - \\
\hline$\rightarrow{ }^{2} T_{2 g}$ & 26229 & 23000 \\
\hline$\rightarrow{ }^{2} T_{1 g}$ & 26621 & - \\
\hline$\rightarrow{ }^{2} E_{g}$ & 27853 & - \\
\hline$\rightarrow{ }^{2} T_{1 g}$ & 31077 & - \\
\hline$\rightarrow{ }^{4} T_{1 g}$ & 33951 & 35000 \\
\hline$\rightarrow{ }^{2} T_{2 g}$ & 35720 & - \\
\hline$\rightarrow{ }^{2} A_{2 g}$ & 37061 & - \\
\hline \multicolumn{3}{|c|}{$\begin{array}{c}{\left[\mathrm{FeF}_{6}\right]^{3-}, \text { ground state }{ }^{6} A_{1 g}[23,28]} \\
\mathrm{B}=835, \mathrm{C}=4800\end{array}$} \\
\hline${ }^{6} A_{1} \rightarrow{ }^{r} T_{1 g}$ & 13700 & 14200 \\
\hline$\rightarrow{ }^{2} T_{2 g}$ & 14843 & - \\
\hline$\rightarrow{ }^{4} T_{2 g}$ & 18938 & 19700 \\
\hline$\rightarrow{ }^{4} A_{1 g}$ & 25050 & 25400 \\
\hline$\rightarrow{ }^{4} E_{g}$ & 25050 & 25400 \\
\hline$\rightarrow{ }^{2} A_{2 g}$ & 25374 & - \\
\hline$\rightarrow{ }^{2} T_{1 g}$ & 25570 & - \\
\hline$\rightarrow{ }^{2} T_{2 g}$ & 27239 & - \\
\hline$\rightarrow{ }^{4} T_{2 g}$ & 28153 & 28800 \\
\hline$\rightarrow{ }^{2} E_{g}$ & 29041 & - \\
\hline$\rightarrow{ }^{4} E_{g}$ & 30895 & 30200 \\
\hline$\rightarrow{ }^{2} T_{1 g}$ & 33298 & - \\
\hline$\rightarrow{ }^{2} A_{1 g}$ & 35251 & - \\
\hline$\rightarrow{ }^{2} T_{2 g}$ & 36649 & - \\
\hline$\rightarrow{ }^{2} E_{g}$ & 36740 & - \\
\hline$\rightarrow{ }^{4} T_{1 g}$ & 36964 & - \\
\hline$\rightarrow{ }^{2} T_{2 g}$ & 38640 & - \\
\hline \multicolumn{3}{|c|}{$\begin{array}{c}{\left[\mathrm{CoF}_{6}\right]^{3-}, \text { ground state }{ }^{5} T_{2 g}[23,29]} \\
\mathrm{B}=787, \mathrm{C}=10000\end{array}$} \\
\hline${ }^{5} T_{2 g} \rightarrow{ }^{1} A_{1 g}$ & 6622 & - \\
\hline$\rightarrow{ }^{3} T_{1 g}$ & 8977 & - \\
\hline$\rightarrow{ }^{3} T_{2 g}$ & 12932 & 11800,14400 \\
\hline$\rightarrow{ }^{5} E_{g}$ & 13114 & - \\
\hline$\rightarrow{ }^{1} T_{1 g}$ & 17656 & - \\
\hline$\rightarrow{ }^{3} T_{1 g}$ & 20129 & - \\
\hline$\rightarrow{ }^{1} E_{g}$ & 20686 & - \\
\hline$\rightarrow{ }^{3} T_{2 g}$ & 21249 & - \\
\hline$\rightarrow{ }^{3} E_{g}$ & 23068 & - \\
\hline$\rightarrow{ }^{3} T_{1 g}$ & 23589 & - \\
\hline$\rightarrow{ }^{1} T_{2 g}$ & 25510 & - \\
\hline$\rightarrow{ }^{3} T_{2 g}$ & 26834 & - \\
\hline
\end{tabular}

the values of optical electron transitions were obtained with high accuracy (in most cases up to a few hundred $\mathrm{cm}^{-1}$ ). The calculated values of $10 \mathrm{Dq}$ are in good agreement with the experimental data with the precision of $200-400 \mathrm{~cm}^{-1}$. An exception is the Jahn-Teller vanadium aquacomplex, but even in this case the difference does not exceed $1000 \mathrm{~cm}^{-1}$, accurate enough.

Comparison of the transition energies in the $d-d-$ spectra, calculated by the ECF method with the

TABLE IV

Calculated and experimental excitation energies $\left(\mathrm{cm}^{-1}\right)$ of transition metal chlorine complexes.

\begin{tabular}{|c|c|c|}
\hline Transition & $\begin{array}{c}\mathrm{E} \\
\text { (theor) }\end{array}$ & $\begin{array}{c}E \\
(\exp )\end{array}$ \\
\hline \multicolumn{3}{|c|}{$\begin{array}{c}{\left[\mathrm{CrCl}_{6}\right]^{3-}, \text { ground state }{ }^{4} A_{2 g}[30,31]} \\
B=918, C=4133\end{array}$} \\
\hline${ }^{4} A_{2 g} \rightarrow{ }^{2} E_{g}$ & 9700 & - \\
\hline$\rightarrow{ }^{2} T_{1 q}^{g}$ & 10148 & - \\
\hline$\rightarrow{ }^{4} T_{2 g}$ & 13206 & 13100 \\
\hline$\rightarrow{ }^{2} T_{2 q}$ & 14796 & - \\
\hline$\rightarrow^{4} T_{1 g}^{2 g}$ & 18272 & 18700 \\
\hline$\rightarrow{ }^{2} A_{1 g}$ & 21206 & - \\
\hline$\rightarrow{ }^{2} T_{2 g}$ & 22810 & - \\
\hline$\rightarrow{ }^{2} T_{1 g}^{2 g}$ & 23086 & - \\
\hline$\rightarrow^{2} E_{\alpha}$ & 24210 & - \\
\hline$\rightarrow^{2} T_{1 g}^{g}$ & 26613 & - \\
\hline$\rightarrow{ }^{4} T_{1 g}$ & 28845 & . \\
\hline
\end{tabular}

$\left[\mathrm{MnCl}_{6}\right]^{3-}$, ground state ${ }^{5} E_{g}[30,32]$ $B=965, C=4450$

$$
\begin{aligned}
{ }^{5} E_{g} & \rightarrow^{3} T_{1 g} \\
& \rightarrow{ }^{1} T_{2 g} \\
& \rightarrow^{1} E_{g} \\
& \rightarrow^{5} T_{2 g} \\
& \rightarrow^{3} E_{g} \\
& \rightarrow^{3} T_{1 g} \\
& \rightarrow^{3} T_{2 g} \\
& \rightarrow^{3} A_{2 g} \\
& \rightarrow^{3} A_{2 g} \\
& \rightarrow^{1} A_{1 g} \\
& \rightarrow^{3} E_{g} \\
& \rightarrow^{1} A_{2 g}
\end{aligned}
$$

5317

16656

16812

17552

19687

20728

21591

23140

23712

24743

26665

29828

$\left[\mathrm{FeCl}_{4}\right]^{-}$, ground state ${ }^{6} A_{1 \mathrm{~g}}[23,31]$

$$
\mathrm{B}=550, \mathrm{C}=220
$$

$$
\begin{aligned}
{ }^{6} A_{1 g} & \rightarrow{ }^{4} T_{1 g} \\
& \rightarrow{ }^{4} T_{2 g} \\
& \rightarrow{ }^{4} A_{1 g}{ }^{4} E_{g} \\
& \rightarrow{ }^{4} A_{1 g},{ }^{4} E_{g} \\
& \rightarrow{ }^{4} T_{2 g} \\
& \rightarrow{ }^{4} E_{g}
\end{aligned}
$$$$
15356
$$$$
16500
$$$$
19192
$$$$
19192
$$$$
20350
$$

22229
15600

16300

18800

18800

20100

22400 
TABLE V

Calculated and experimental excitation energies $\left(\mathrm{cm}^{-1}\right)$ of transition metal aquacomplexes.

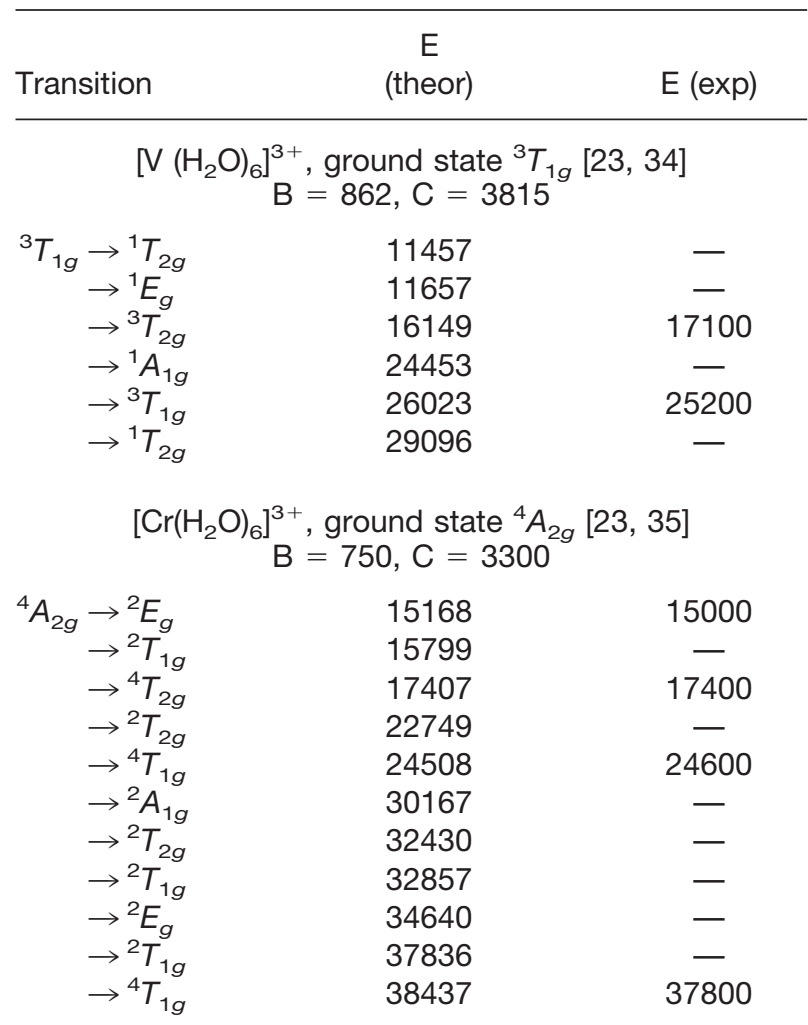

$\left[\mathrm{Fe}\left(\mathrm{H}_{2} \mathrm{O}\right)_{6}\right]^{3+}$, ground state ${ }^{6} A_{1 g}[23,36]$

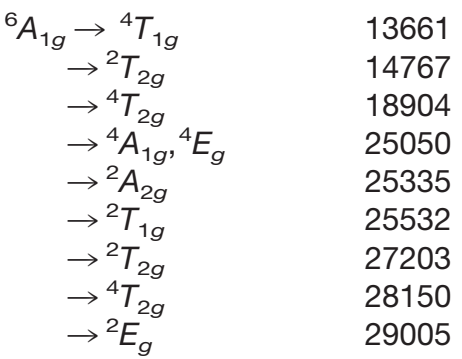

12600

-

18500

24300,24600

-

-

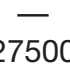

$\left[\mathrm{Co}\left(\mathrm{H}_{2} \mathrm{O}\right)_{6}\right]^{3+}$, ground state ${ }^{1} A_{19}[23,37]$ $B=615, C=3085$

$\begin{array}{rlr}{ }^{1} A_{1 g} & { }^{3} T_{1 g} & 9649 \\ & \rightarrow{ }^{5} T_{2 g} & 10347 \\ & \rightarrow^{3} T_{2 g} & 13486 \\ & \rightarrow^{1} T_{1 g} & 16227 \\ & \rightarrow^{1} E_{g} & 19213 \\ & \rightarrow^{1} T_{2 g} & 24051 \\ & \rightarrow^{3} T_{1 g} & 25928 \\ & \rightarrow^{3} T_{2 g} & 26718 \\ & \rightarrow^{3} E_{g} & 28474 \\ & \rightarrow^{5} E_{g} & 28503 \\ & { }^{3} T_{1 g} & 28990\end{array}$

parameterization cited in Table VII, with the experimental absorption band energies demonstrates that calculation with the fitted parameters leads to results that are in good agreement with experimental data. The divergences are in the range of 1000 $\mathrm{cm}^{-1}$ except for cyanocomplexes. The calculated transition energies in the latter case differ from the experimental energies by up to $2000 \mathrm{~cm}^{-1}$. However, two experimental works concerning iron(III) hexacyanide $[23,24]$ give for the same states energy values differing by about $1000 \mathrm{~cm}^{-1}$. Thus, one may conclude that the accuracy of the ECF method with the given parameterization is comparable with the accuracy of the spectral methods themselves.

Figures given in Table VII concerning the differences of the metal-donor atom separations between the complexes of di- and trivalent cations show some regularities. In all cases, with the exception of the pair of iron hexacyanocomplexes where the $\mathrm{Fe}-\mathrm{C}$ distance increases by $0.037 \AA$, the metal-

TABLE VI

Calculated and experimental excitation energies $\left(\mathrm{cm}^{-1}\right)$ of transition metal amminocomplexes.

\begin{tabular}{ccc}
\hline & $E$ & $E$ \\
Transition & (theor) & (exp)
\end{tabular}

$\left[\mathrm{Cr}\left(\mathrm{NH}_{3}\right)_{6}\right]^{3+}$, ground state ${ }^{4} A_{2 g}[23,38]$

\begin{tabular}{|c|c|c|}
\hline$g \rightarrow{ }^{2} E_{g}$ & 15283 & 15300 \\
\hline$\rightarrow{ }^{2} T_{1 g}$ & 15882 & - \\
\hline$\rightarrow{ }^{4} T_{2 g}$ & 21538 & 1550 \\
\hline$\rightarrow{ }^{2} T_{2 q}$ & 23398 & \\
\hline$\rightarrow{ }^{4} T_{1 g}$ & 28984 & 28500 \\
\hline$\rightarrow{ }^{2} A_{1 g}$ & 34298 & \\
\hline$\rightarrow{ }^{2} T_{2 g}$ & 36645 & \\
\hline$\rightarrow{ }^{2} T_{1 g}^{2 g}$ & 36986 & \\
\hline$\rightarrow^{2} E_{g}$ & 38771 & \\
\hline
\end{tabular}

$\left[\mathrm{Co}\left(\mathrm{NH}_{3}\right)_{6}\right]^{3+}$, ground state ${ }^{1} A_{19}[23,39]$

$$
\mathrm{B}=615, \mathrm{C}=3085
$$

$\begin{array}{rlr}{ }^{1} A_{1 g} & \rightarrow{ }^{3} T_{1 g} & 14287 \\ & \rightarrow{ }^{3} T_{2} & 18350 \\ & \rightarrow{ }^{5} T_{2 g} & 19559 \\ & \rightarrow{ }^{1} T_{1 g} & 20815 \\ & \rightarrow^{1} E_{g} & 24046 \\ & \rightarrow^{1} E_{g} & 24046 \\ & \rightarrow^{1} T_{2 g} & 29075 \\ & \rightarrow^{3} T_{1 g} & 35148 \\ & \rightarrow^{3} T_{2 g} & 35919 \\ & \rightarrow^{3} T_{2 g} & 35919 \\ & \rightarrow^{3} E_{g} & 37759 \\ & \rightarrow{ }^{3} T_{1 g} & 38354\end{array}$

13000

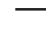

21200

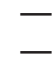

29550

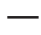


TABLE VII

Calculated and experimental excitation energies $\left(\mathrm{cm}^{-1}\right)$ of transition metal cyanocomplexes.

\begin{tabular}{lcc}
\hline & $E$ & $E$ \\
Transition & (theor) & (exp) \\
\hline
\end{tabular}

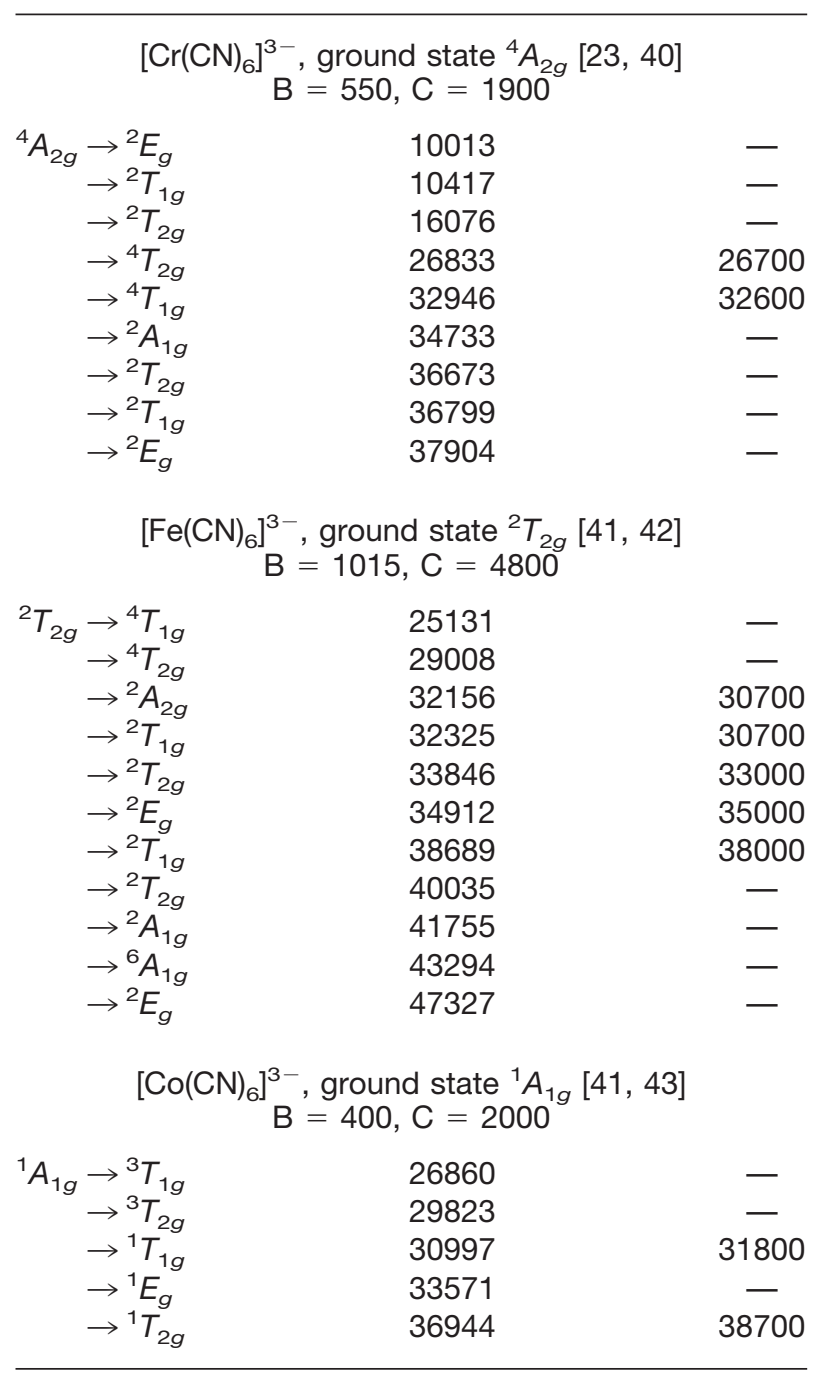

donor atom distances decrease by $0.1-0.3 \AA$. However, in the pair of hexaaquacomplexes of cobalt an essentially smaller difference $(0.011 \AA)$ is observed. We also note that for the majority of cations the complexes of the trivalent ones possess total spin values that are smaller than those for their divalent counterparts. If a complex of a divalent cation has a low spin, the corresponding complex of a trivalent cations also has a low spin and vice versa. The only exception is provided by the aquacomplexes of Co. This anomaly in the experimental data can be reflected in the ECF calculations only if the regularity in the $\beta^{M-L}$ parameter variation along the row is broken. In the case of the cobalt hexaaquacomplexes pair the difference of $\beta^{M-L}$ values is abnormally large (0.7, essentially higher than the difference typical for all other complexes: 0.2-0.4). This fact requires that we perfect our approach, for example, by adjusting a larger number of parameters. Nevertheless, in the case of the iron hexacyanocomplexes the difference of $\beta^{M-L}$ appears to be regular despite the anomalous difference of the metal-donor atom separation.

In the present work the ECF method [14] is employed for calculations of the complexes of trivalent ions of the first row transition metals. It is demonstrated that the description of the $d$-d-excitation spectra of the trivalent ion complexes is possible with the same high accuracy as in the case of the complexes of respective divalent ions, provided the parameters of the resonance interactions between the $d$-shell and ligands are respectively adjusted.

\section{ACKNOWLEDGMENTS}

This work was partially supported by RFBR Grant 99-03-33176, the Federal Task Programm of Russia "Integracia" Grant A0078, and the special RAS Grant Program 6 for younger scientists by Grant 120. The work has been performed using the Net Laboratory system [44] of access to quantum chemical software sponsored by the RFBR through Grant 01-07-90383.

\section{REFERENCES}

1. Zerner, M. C.; Bacon, A. D. Theor Chem Acta 1979, 53, 21.

2. Zerner, M. C.; Loew, G. H.; Kirchner, R. F.; Mueller-Westerhoff, U. T. J Am Chem Soc 1980, 102, 589.

3. Edwards, W. D.; Weiner, B.; Zerner, M. C. J Phys Chem 1988, $110,6188$.

4. Edwards, W. D.; Weiner, B.; Zerner, M. C. J Am Chem Soc 1986, 108, 2196

5. Kotzian, M.; Rösch, N.; Schröder, H.; Zerner, M. C. J Am Chem Soc 1989, 111, 7687.

6. Newton, J. E.; Hall, M. B. Inorg Chem 1984, 23, 4627.

7. Newton, J. E.; Hall, M. B. Inorg Chem 1985, 24, 2573.

8. Pierloot, K.; Vanquickenborn, L. G. J Chem Phys 1990, 93, 4154.

9. Rosi, M.; Bauschlicher, C. W.; Langhoff, S. R.; Partrige, H. J Phys Chem 1990, 94, 8656.

10. Morokuma, K. Inorg Chem 1990, 29, 3110.

11. Johansen, H.; Andersen, N. K. Mol Phys 1986, 58, 965.

12. Shashkin, S. Y.; Goddard, W. A. Phys Rev B 1986, 33, 153.

13. Janssen, G. J. M.; Nieuwpoort, W. C. Int J Quantum Chem Symp 1988, 22, 679 . 
14. Soudackov, A. V.; Tchougréeff, A. L.; Misurkin, I. A. Theor Chem Acta 1992, 83, 389.

15. Bersuker, I. B. Electronic Structure and Properties of Transition Metal Complexes; Nauka: Moscow, 1986 (in Russian).

16. Jørgensen, C. K. Modern Aspects of Ligand Field Theory; North-Holland: Amsterdam, 1971.

17. Löwdin, P.-O. Perturbation Theory and its Application in Quantum Mechanics; Wiley: New York, 1966.

18. McWeeny, R. Methods of Molecular Quantum Mechanics (Series in Theoretical Chemistry); Academic Press: New York, 1992.

19. Soudackov, A. V.; Tchougréeff, A. L.; Misurkin, I. A. Int J Quantum Chem 1996, 58, 161.

20. Soudackov, A. V.; Tchougréeff, A. L.; Misurkin, I. A. Russ J Phys Chem 1994, 68, 1256.

21. Soudackov, A. V.; Tchougréeff, A. L.; Misurkin, I. A. Russ J Phys Chem 1994, 68, 1264.

22. Tokmachev, A. N.; Tchougréeff, A. L. Chem Phys Rep 1999, 18,80 .

23. Jørgensen, C. K. Adv Chem Phys 1963, 5, 33.

24. Lever, A. B. P. Inorganic Electronic Spectroscopy; Elsevier: Amsterdam, 1984.

25. Böhm, M. C.; Gleiter, R. Theor Chim Acta 1981, 59, 127.

26. Alter, E.; Hoppe, R. Z Anorg Allg Chem 1975, 412, 110.
27. Allen, G. C.; El-Sharkawy, G. A. M.; Warren, K. C. Inorg Chem 1971, 11, 2538.

28. Bode, V. H.; Voss, E. Z Anorg Allg Chem 1957, 290, 1.

29. Fleischer, T.; Hoppe, R. J Fluor Chem 1982, 19, 529.

30. Hatfield, W. E.; Fay, R. C.; Pfluger, C. E.; Piper, T. S. J Am Chem Soc 1963, 85, 265.

31. Friedrich, G.; Fink, H.; Seifert, H. J. Z Anorg Allg Chem 1987, $548,141$.

32. Lalanette, R. A.; Elliot, N.; Bernal, I. J Cryst Mol Struct 1972, $2,143$.

33. Meyer, V. G. Z Anorg Allg Chem 1977, 436, 87.

34. Cotton, F. A.; Fair, C. K.; Lewis, G. E. J Am Chem Soc 1984, 106, 5319.

35. Epple, M.; Massa, W. Z Anorg Allg Chem 1978, 444, 47.

36. Hair, N. J.; Beattie, J. K. Inorg Chem 1977, 16, 245.

37. Lynton, H.; Siew, P. Y. Can J Chem 1973, 51, 227.

38. Clegg, W. Acta Cryst B 1976, 32, 2907.

39. Schröder, D. R.; Jacobson, R. A. Inorg Chem 1973, 12, 210.

40. Jagner, S.; Ljungström, E.; Vannerberg, N. G. Acta Chem Scand 1974, 28, 623.

41. Alexander, J. J.; Gray, H. B. J Am Chem Soc 1968, 90, 4260.

42. Vannerberg, N. G. J Am Chem Soc 1972, 26, 2863.

43. Swanson, B. I.; Ryan, R. R. Inorg Chem 1973, 12, 283.

44. http://qcc.ru/ netlab. 